\title{
Using hybrid topological-spin qubit systems for two-qubit-spin gates
}

\author{
Martin Leijnse and Karsten Flensberg \\ Center for Quantum Devices, Niels Bohr Institute, \\ University of Copenhagen, 2100 Copenhagen Ø, Denmark
}

\begin{abstract}
We investigate a hybrid quantum system involving spin qubits, based on the spins of electrons confined in quantum dots, and topological qubits, based on Majorana fermions. In such a system, gated control of the charge on the quantum dots allows transfer of quantum information between the spin and topological qubits, and the topological system can be used to facilitate transfer of spin qubits between spatially separated quantum dots and to initialize entangled spin-qubit pairs. Here, we show that the coupling to the topological system also makes it possible to perform entangling two-qubit gates on spatially separated spin qubits. The two-qubit gates are based on a combination of topologically protected braiding operations, gate-controlled charge transfer between the dots and edge Majorana modes, and measurements of the state of the topological qubits.
\end{abstract}

PACS numbers: 03.67.Lx, 85.35.Gv, 74.45.+c, 74.20.Mn,

\section{INTRODUCTION.}

Spin qubits defined in quantum dots ${ }^{1}$ is a promising candidate for quantum information processing because of long coherence times and efficient implementation of one- and two-qubit gates, as demonstrated in a number of recent experiments $\mathrm{s}^{2}-\frac{5}{2}$. Single-qubit rotations can be implemented with time-dependent magnetic fields ${ }^{4}$, or with electric fields utilizing the spin-orbit coupling ${ }^{5}$. Two-qubit gates can be implemented by controlling the exchange interaction in a double dot $\underline{1}$, which is accomplished through gated control of the inter-dot tunnel coupling. The exchange interaction can also be used for single-qubit rotations, if the qubit is defined in some twodimensional subspace of the two double dot spins ${ }^{3.6}, \underline{3}$, e.g., the singlet and one of the triplets. Readout can be done via spin-to-charge conversion, where one spin qubit state is prohibited from tunneling out of the dot, either by a Zeeman splitting $\underline{\underline{7}}$ (in a single-spin qubit) or by Pauli spin-blockade ${ }^{3}$ (in a singlet-triplet qubit).

One problem with spin qubits is the limited range of the exchange interaction, causing problems with scaling to larger (many-qubit) systems ${ }^{8}$. Similar problems are encountered in many other qubit realizations since it is hard to achieve long coherence times for a type of qubit which is sensitive to long-ranged interactions. Therefore, much theoretical and experimental work has been aimed at designing quantum systems which can facilitate coupling between two (or more) spatially separated qubits, thus acting as a quantum bus for the primary qubits. An early example is the proposal to use phonons to couple qubits defined in trapped ions ${ }^{9}$. More recently, coupling of superconducting phase $\frac{10}{}$ and charge $\frac{11}{11}$ qubits via a microwave field (circuit quantum electrodynamics) has been achieved experimentally. The same principles have been considered for spin qubits $\stackrel{12-14}{2}$, but this case is experimentally more problematic because of the rather weak coupling between the electron spin and the cavity field. Other theoretical suggestions involve e.g., coupling to extended spin chains 15 .

In this work we study the possibility of using a topo- logical superconductor (TS) as a quantum bus for spin qubits. A TS is a superconductor with $p$-wave-type pairing, which has been argued to be realized e.g., in $\mathrm{Sr}_{2} \mathrm{RuO}_{4} \stackrel{16}{ }$. Recently it was shown that $p$-wave type pairing may occur in topological insulators $\frac{17}{}$ and strong spin-orbit semiconductors $18-21$ with induced magnetism and superconductivity. Perhaps the most spectacular aspect of TS is the Majorana fermion ${ }^{22}$ (MF) quasiparticles which form in vortices and at edges, first discussed in the context of the $\nu=5 / 2$ fractional quantum Hall state $^{23}$. Basically, a MF is "half of a Dirac fermion" and two MFs together form one fermionic degree of freedom, which may be highly nonlocal if the MFs are spatially separated. This non-locality offers protection from many types of decoherence. In topological quantum computation schemes 24,25 , these non-local degrees of freedom are manipulated by braiding operations, i.e., by physical exchange of the associated local quasiparticle excitations. This is possible because MFs satisfy non-abelian (noncommutative) statistics 26.27 . A major problem for topological quantum computation is that the exchange statistics of MFs does not allow for universal quantum computation through braiding operations alone ${ }^{25}$. We showed in a recent work ${ }^{28}$ that quantum information can be coherently transferred between topological qubits (based on MFs) and normal spin qubits. This allows using topological qubits as quantum memories in a spin-based quantum computer, or, alternatively, to use spin-qubits to prepare arbitrary topological qubit states. Other works have considered interfaces between topological qubits and conventional superconducting qubits $\frac{29}{21}$ or charge qubits defined in a double quantum $\operatorname{dot}^{32}$.

Here we show that a TS can be used to directly perform entangling two-qubit gates on spatially separated spin qubits. The two-qubit gates consist exclusively of protected braiding operations acting on a type of overlapping topological two-qubit states, and only the transfer of quantum information between the spin and topological qubits remain (partially) unprotected. Furthermore, unlike pulsed exchange interactions which require careful timing, such braiding-based two-qubit gates leave 


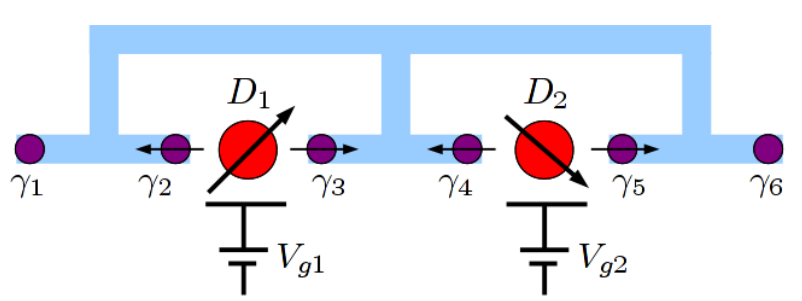

FIG. 1: (Color online) Sketch of setup. The electrostatic potentials on the quantum dots $D_{1}$ and $D_{2}$ are controlled by the gate voltages $V_{g 1}$ and $V_{g 2}$. This allows control of their occupation, which can be either empty (zero electrons on the dot) or filled (one electron on the dot), with a filled dot acting as a spin qubit. The 1D topological superconducting wires host MFs described by the operators $\gamma_{1}, \ldots, \gamma_{6}$ at the edges. The T-junctions and additional wire allow exchange (braiding) of the MFs. Arrows indicate the anti-parallel spin polarization of the two MFs which couple to each dot. As described in the main text, this setup allows two-qubit gates to be performed on the spin qubits on $D_{1}$ and $D_{2}$, which can be arbitrarily far apart, as long as the MFs can be initialized, braided and measured.

no room for continuous errors (two particles cannot be partially exchanged and an electron cannot be partially transferred between the dots and the TS).

\section{HYBRID SPIN-TOPOLOGICAL QUBIT SYSTEM}

A sketch of the hybrid spin-topological quantum system is shown in Fig. 1 Here the TS consists of onedimensional (1D) wires 20.21 , connected to allow for particle exchange, but the results in the paper are not restricted to this specific geometry. To keep the discussion as simple as possible, we focus throughout the paper on a rather simple model. The assumptions underlying this model, as well as the effect of deviations from the model, are discussed in Sect. $\mathbf{V}$ The Hamiltonian is then given by $H_{1}+H_{2}$, where

$$
\begin{aligned}
H_{i} & =\epsilon_{i} \sum_{\sigma} n_{i \sigma}+U_{i} n_{i \uparrow} n_{i \downarrow} \\
& +\gamma_{2 i}\left(\lambda_{2 i} d_{i \uparrow}-\lambda_{2 i}^{*} d_{i \uparrow}^{\dagger}\right) \\
& +\gamma_{2 i+1}\left(\lambda_{2 i+1} d_{i \downarrow}-\lambda_{2 i+1}^{*} d_{i \downarrow}^{\dagger}\right) .
\end{aligned}
$$

The first line describes the single-level quantum dot $D_{i}$ with occupation number $n_{i \sigma}=d_{i \sigma}^{\dagger} d_{i \sigma}$ and onsite energy $\epsilon_{i}$, which can be controlled with the gate voltage, $\epsilon_{i} \propto-V_{g i} . U_{i}$ is the Coulomb charging energy, which is typically large compared with the other relevant energy scales and we take the limit $U_{i} \rightarrow \infty$. We have assumed the onsite energies to be spin-degenerate.

The second and third lines in Eq. (11) describe tunneling between the dot and the TS, see also e.g., Refs 28,33,34. We here consider only dot energies below the supercon- ducting gap, $\left|\epsilon_{i}\right| \ll \Delta$, where the tunneling is dominated by coupling to the zero-energy edge MFs which are described by the $\gamma$ operators. A MF is always spinpolarized in the sense that, for a given tunnel junction, there exist a spin-quantization axis along which it is only possible to tunnel into or out of the state with electrons with a specific spin projection. In Eq. (1) we have assumed this spin polarization to be anti-parallel for the two MFs which couple to a given dot, i.e, for $\gamma_{2 i}$ and $\gamma_{2 i+1}$ (this assumption is motivated in Sect. $\mathrm{V}$ ). We choose the quantization axis for the dot spin such that the polarization direction associated with the left (right) tunnel junction is spin up (spin down). This is why in Eq. (10), $\gamma_{2 i}\left(\gamma_{2 i+1}\right)$ only couples to the spin-up (spin-down) dot electron operators.

The MF operators are hermitian, $\gamma_{i}^{\dagger}=\gamma_{i}$, and we assume them to be normalized, $\gamma_{i}^{2}=1$. Thus, $\gamma_{i}^{\dagger} \gamma_{i} \equiv 1$ and it is not possible to construct a MF number operator, or to count the occupation of a Majorana mode. However, two Majoranas can be combined to form one ordinary fermion and we choose to pair neighboring MFs, such that

$$
\begin{aligned}
\gamma_{2 i-1} & =f_{i}+f_{i}^{\dagger}, \\
\gamma_{2 i} & =i\left(f_{i}^{\dagger}-f_{i}\right),
\end{aligned}
$$

where $f_{i}^{\dagger}$ creates a fermion and $f_{i}^{\dagger} f_{i}=n_{i}=0,1$ counts the occupation of the corresponding state. Thus, two MFs together form a single fermionic state which can be either empty or filled and the 6 MFs in our system span a $2^{3}$-dimensional space. We assume vanishing coupling between the different MFs, which is satisfied when they are well separated on a length scale set by the effective superconducting coherence length in the TS. Then the MFs do not appear in the Hamiltonian, except for in the tunnel term. Because of the anti-parallel spin polarization, there is also no effective coupling between $\gamma_{2 i}$ and $\gamma_{2 i+1}$ via the quantum dots, and all the 8 fermionic states are therefore degenerate.

Below we discuss in detail the different quantum computational operations which can be performed with the system in Fig. 1] but we want to already now give an intuitive explanation of the central aspect, namely the capability to transfer quantum information from the spin of an electron in one of the dots to topological information encoded in the MFs. Let us restrict our attention to $D_{1}$ and the MFs 1-4 and assume that the level of $D_{1}$ is initially far below the chemical potential of the superconductor, such that the dot is occupied by a single electron (spin qubit). If we now adjust the gate voltage to bring up the dot level, it will eventually empty and the electron will tunnel into the TS (many electrons may tunnel back and forth, but there will be a net transfer of one electron from the dot to the TS). If the dot level is kept well inside the superconducting gap, this can only happen via tunneling into the MFs 2 and 3. Because of the anti-parallel spin polarizations of these MFs, the electron must split into a spin up and a spin down component (along the 
quantization axis of the MFs polarization), each component tunneling into opposite sides of the TS. Adding a charge to the TS to the left (right) side of $D_{1}$ corresponds to flipping the fermionic occupation number $n_{1}$ $\left(n_{2}\right)$. Thus, by adding a fractional charge to each side, where the fraction depends on the spin projection of the electron, we rotate each of the fermionic number states by an amount which depends on the electron spin. This allows translation from spin quantum information into topological quantum information. In the same way, we can transfer topological quantum information into spin quantum information.

\section{BASIC COMPUTATIONAL AND TRANSFER OPERATIONS}

The computational operations discussed in this paper rely on the ability to carry out a number of different operations on the quantum dots and MFs. We list these operations here and discuss them in more detail below.

1. $P_{F}^{i}$ : Fill (an initially empty) dot $i$ with an electron from the TS by a gate sweep which shifts $\epsilon_{i}$ from negative to positive.

2. $P_{E}^{i}$ : Empty (an initially occupied) dot $i$, transferring the electron to the TS, by a gate sweep which shifts $\epsilon_{i}$ from positive to negative.

3. $B_{i}$ : Braid (exchange) MFs $i$ and $i+1$.

4. $I_{i}$ : Initialize the fermion number states $n_{i}$ (the ability to produce $n_{i}=0$ is sufficient).

5. $M_{i}$ : Measure the fermionic occupation numbers $n_{i}$.

1. It was shown $\operatorname{in}^{28}$ that if $\operatorname{dot} i$ is initially empty, a gate sweep which takes $\epsilon_{i}$ from $\epsilon_{i} /\left|\bar{\lambda}_{i}\right| \ll-1$ to $\epsilon_{i} /\left|\bar{\lambda}_{i}\right| \gg$ 1 has the same effect (up to an overall phase) as acting on the state of the total system with the operator

$$
P_{F}^{i}=\frac{1}{\sqrt{2}}\left(\gamma_{2 i} d_{i \uparrow}^{\dagger}+\gamma_{2 i+1} d_{i \downarrow}^{\dagger}\right),
$$

where we have assumed $\bar{\lambda}_{i}=\lambda_{2 i}=\lambda_{2 i+1}$ (a relative phase between $\lambda_{2 i}$ and $\lambda_{2 i+1}$, introducing a phase in one of the terms in Eq. (4), does not significantly alter the results below, but we exclude it here for simplicity). The requirement for Eq. (4) to be valid is that the gate sweep is done adiabatically with respect to the tunnel coupling $\bar{\lambda}_{i}$, so that the system remains in the ground state as the dot is emptied.

2. Similar to above, if $\operatorname{dot} i$ is initially filled with an electron, an adiabatic gate sweep which takes $\epsilon_{i}$ from $\epsilon_{i} /\left|\bar{\lambda}_{i}\right| \gg 1$ to $\epsilon_{i} /\left|\bar{\lambda}_{i}\right| \ll-1$ has the same effect (up to an overall phase) as acting with the operator

$$
P_{E}^{i}=N\left(\gamma_{2 i} d_{i \uparrow}+\gamma_{2 i+1} d_{i \downarrow}\right) .
$$

There is one complication here: Eq. (5) assumes that the dot is successfully emptied, which is, in fact, not guaranteed even if the gate sweep is done adiabatically. The reason is that, due to the spin degeneracy, the dimension of the Hilbert space with an empty dot is smaller than that with a full dot. Therefore, the ground state manifold with a full dot must have a component which is disconnected from the smaller ground state manifold with an empty dot. Equation (5) should be interpreted as the operation of making the adiabatic gate sweep, followed by a measurement of the charge on the dot, assuming that this charge measurement shows the dot to be empty. However, this is not a truly restricting assumption: If the charge measurement shows that emptying the dot was not successful, the original spin qubit can be recovered and the transfer attempted until successful, see Ref. 28 for details. Note that, because of the collapse of the wave function, resulting from this charge measurement, the normalization $N$ is an operator (i.e., the correct normalization factor depends on the state upon which $P_{E}$ is acting).

3. The effect of physically exchanging MFs $i$ and $i+1$ is described by the operator ${ }^{27}$

$$
B_{i}=\frac{1}{\sqrt{2}}\left(1+\gamma_{i} \gamma_{i+1}\right) .
$$

It was recently discussed that MFs can be transported and exchanged in networks of one-dimensional wires using "keyboard gates" 35 . There are also alternatives which accomplish the same thing as braiding operations, without actual physical exchange of $\mathrm{MFs}_{\mathrm{s}} 36,37$.

4. If a section of the wire is tuned from a nontopological to a topological phase, e.g., using gate voltages, one MF will appear in each end of the created topological region. Since such an operation does not change the parity of the fermion number in the superconductor, the associated fermion state must be empty, $n_{i}=0$, see e.g. $\frac{35}{5}$.

5. If the MFs $2 i-1$ and $2 i$ are brought into proximity with each other, introducing a coupling $\xi$ between them, this gives rise to a term $i \xi \gamma_{2 i-1} \gamma_{2 i} / 2=\xi n_{i}$ in the Hamiltonian, breaking the degeneracy associated with occupying the fermionic state which can then be measured 24 . The occupation numbers could also be measured e.g., using interferometry 38 or by coupling to conventional superconducting qubits 29 .

\section{TWO-QUBIT GATES WITH SPATIALLY SEPARATED SPIN QUBITS}

In a slightly simpler setup than the one sketched in Fig. 11 we showed in Ref. 28 that the operations described above can be used to:

i Transfer a spin qubit on $D_{i}$ into a topological qubit defined within the subspace of four MFs (using the operations $\left.P_{E}^{i} I_{i} I_{i+1}\right)$. 
ii Transfer a topological qubit into a spin qubit $\left(M_{i} P_{F}^{i} I_{i} I_{i+1}\right)$.

iii Transfer a spin qubit from one dot to the other $\left(M_{1} P_{F}^{2} P_{E}^{1} I_{2} I_{1}\right)$

iv Initialize the two dot spins in a maximally entangled Bell state $\left(M_{1} P_{F}^{2} P_{F}^{1} I_{2} I_{1}\right)$.

This can be accomplished in a setup with only fours MFs and does not require the ability to braid MFs. We now show that, with a six-MF setup as in Fig. 1, and with the capability to braid the MFs, one can in addition perform non-trivial two-qubit gates on spin qubits on dots 1 and 2 .

The combined state of the two dots is $\left|D_{1} D_{2}\right\rangle_{D}$, where $D_{i}=0, \uparrow, \downarrow$, corresponding to dot $i$ being empty or filled with a spin-up or spin-down electron. We characterize the Majorana system in terms of the number states $\left|n_{1} n_{2} n_{3}\right\rangle_{M}$. Since braiding operations cannot change the parity of the total fermion number, four MFs are needed to form one topological qubit ${ }^{39}$, for example within the subspace with even total fermion number parity. It is then natural to use eight MFs to make a topological twoqubit system. However, it turns out that it is not possible to entangle two such topological qubits using only braiding operations 39 . Therefore, we cannot simply use the results of Ref. 28 to transfer two spin qubits to two separate topological qubits, entangle these and then transfer the information back into the spin system.

Instead, we follow an idea by Georgiev $\underline{40}$ and note that six MFs with fixed total parity is sufficient to define a type of effective two-qubit system. If we again pick the subspace with even total parity we can define the twoqubit states as:

$$
\begin{aligned}
|\overline{0} \overline{0}\rangle_{Q} & =|000\rangle_{M}, \\
|\overline{0} \overline{1}\rangle_{Q} & =|011\rangle_{M}, \\
|\overline{1} \overline{0}\rangle_{Q} & =|110\rangle_{M}, \\
|\overline{1} \overline{1}\rangle_{Q} & =|101\rangle_{M} .
\end{aligned}
$$

Thus, the individual qubit states can be read off from $n_{1}$ and $n_{3}$, while the fermion occupation $n_{2}$ is "shared" between the two qubits and used to balance the fermion number parity.

In the basis $\left\{|\overline{0} \overline{0}\rangle_{Q},|\overline{0} \overline{1}\rangle_{Q},|\overline{1} \overline{0}\rangle_{Q},|\overline{1} \overline{1}\rangle_{Q}\right\}$, a straightforward evaluation of the matrix elements of Eq. (6) shows that, up to overall phases, the braid matrices are given by

$$
\begin{aligned}
B_{1} & =\left(\begin{array}{llll}
i & 0 & 0 & 0 \\
0 & i & 0 & 0 \\
0 & 0 & 1 & 0 \\
0 & 0 & 0 & 1
\end{array}\right), B_{2}=\frac{1}{\sqrt{2}}\left(\begin{array}{llll}
1 & 0 & i & 0 \\
0 & 1 & 0 & i \\
i & 0 & 1 & 0 \\
0 & i & 0 & 1
\end{array}\right) \\
B_{3} & =\left(\begin{array}{llll}
i & 0 & 0 & 0 \\
0 & 1 & 0 & 0 \\
0 & 0 & 1 & 0 \\
0 & 0 & 0 & i
\end{array}\right), B_{4}=\frac{1}{\sqrt{2}}\left(\begin{array}{cccc}
1 & i & 0 & 0 \\
i & 1 & 0 & 0 \\
0 & 0 & 1 & -i \\
0 & 0 & -i & 1
\end{array}\right) \\
B_{5} & =\left(\begin{array}{llll}
i & 0 & 0 & 0 \\
0 & 1 & 0 & 0 \\
0 & 0 & i & 0 \\
0 & 0 & 0 & 1
\end{array}\right)
\end{aligned}
$$

To demonstrate how to perform two-qubit gates on the spin qubits on $D_{1}$ and $D_{2}$, we start from an initial state, $|i\rangle$, with one spin qubit in each dot and each fermionic number state of the TS initialized to zero:

$|i\rangle=\left(\alpha_{1}|\uparrow\rangle_{D_{1}}+\beta_{1}|\downarrow\rangle_{D_{1}}\right)\left(\alpha_{2}|\uparrow\rangle_{D_{2}}+\beta_{2}|\downarrow\rangle_{D_{2}}\right)|\overline{0} \overline{0}\rangle_{Q}$,

where we used the rather obvious notation $\left|D_{1}\right\rangle_{D_{1}}\left|D_{2}\right\rangle_{D_{2}}=\left|D_{1} D_{2}\right\rangle_{D}$. The first step is to empty both dots via adiabatic gate sweeps of $V_{g 1}$ and $V_{g 2}$, which gives the state

$$
\begin{aligned}
P_{E}^{1} P_{E}^{2}|i\rangle & =|00\rangle_{D}\left(-\alpha_{1} \alpha_{2}|\overline{1} \overline{0}\rangle_{Q}+i \alpha_{1} \beta_{2}|\overline{1} \overline{1}\rangle_{Q}\right. \\
& \left.+i \beta_{1} \alpha_{2}|\overline{0} \overline{0}\rangle_{Q}+\beta_{1} \beta_{2}|\overline{0} \overline{1}\rangle_{Q}\right) \\
& =|00\rangle_{D}\left(\alpha_{1}|\overline{1}\rangle_{Q_{1}}-i \beta_{1}|\overline{0}\rangle_{Q_{1}}\right) \\
& \times\left(-\alpha_{2}|\overline{0}\rangle_{Q_{2}}+i \beta_{2}|\overline{1}\rangle_{Q_{2}}\right) .
\end{aligned}
$$

Thus we see that emptying the dots has the effect of transferring the spin qubits into topological qubits: Up to phase factors $|\uparrow\rangle_{D_{1}} \rightarrow|\overline{1}\rangle_{Q_{1}},|\downarrow\rangle_{D_{1}} \rightarrow|\overline{0}\rangle_{Q_{1}}$, and $|\uparrow\rangle_{D_{2}} \rightarrow|\overline{0}\rangle_{Q_{2}},|\downarrow\rangle_{D_{2}} \rightarrow|\overline{1}\rangle_{Q_{2}}$. Note, however, that interpreting the factorized form of the topological twoqubit states $\left(\left|\bar{Q}_{1}\right\rangle_{Q_{1}}\left|\bar{Q}_{2}\right\rangle_{Q_{2}}=\left|\bar{Q}_{1} \bar{Q}_{2}\right\rangle_{Q}\right)$ in the second equality requires some care. The occupation number $n_{2}$, which is suppressed in this notation, depends on both $n_{1}$ and $n_{3}$ due to the constraint of even total parity.

If we now fill the two dots again (applying $P_{F}^{1} P_{F}^{2}$ ), an entangled four-qubit state results, involving the two spin qubits and the two topological qubits. To keep the formulas short, we present the result for the case where both dots were initially initialized in the spin up state, i.e., $\left|i^{\prime}\right\rangle=|\uparrow \uparrow\rangle_{D}|\overline{0} \overline{0}\rangle_{Q}$. From Eq. (13) we see that emptying both dots gives

$$
|j\rangle=P_{E}^{1} P_{E}^{2}\left|i^{\prime}\right\rangle=-|00\rangle_{D}|\overline{1} \overline{0}\rangle_{Q} .
$$

This result is easy to understand: Since both spin-up electrons must tunnel to the left, they change the fermion occupation numbers $n_{1}$ and $n_{2}$ from 0 to 1 . Filling both dots then gives

$$
\begin{aligned}
P_{F}^{1} P_{F}^{2}|j\rangle & =-\frac{1}{2}\left(|\uparrow \uparrow\rangle_{D}|\overline{0} \overline{0}\rangle_{Q}+|\downarrow \downarrow\rangle_{D}|\overline{1} \overline{1}\rangle_{Q}\right. \\
& \left.-i|\downarrow \uparrow\rangle_{D}|\overline{1} \overline{0}\rangle_{Q}-i|\uparrow \downarrow\rangle_{D}|\overline{0} \overline{1}\rangle_{Q}\right) .
\end{aligned}
$$


We can get back the spin qubits by collapsing the wavefunction of the topological qubits, which is accomplished by measuring the topological qubits $Q_{1}$ and $Q_{2}$ (by measuring $n_{1}$ and $n_{3}$ ). This gives four possible outcomes with equal probability, each of which describes two spin qubits. As is seen from Eq. (16), depending on the measured values of $n_{1}$ and $n_{3}$, one or both spin qubits may have been flipped compared with the initial state (note that compared with $\left|i^{\prime}\right\rangle$, a flip of the spin on dot 1 (2) is always accompanied by a change of $\left.n_{1}\left(n_{3}\right)\right)$. However, the spin qubits are still always in a product state, also for a general initial state like $|i\rangle$. This result demonstrates that two spin qubits can be simultaneously saved in the overlapping topological two-qubit system and then recovered. However, it also shows that transfer operations alone are insufficient for performing entangling two-qubit gates on the spin qubits.

What is needed is to entangle the topological qubits $Q_{1}$ and $Q_{2}$, before transferring them back into the spin system. Fortunately, braiding operations can create entangled states in the overlapping two-qubit basis (7)-(10). From Eq. (11) it is clear that the operations $B_{2}$ and $B_{4}$ have the effect of creating an entangled state when acting on a two-qubit product state. These are the braiding operations which involve one of the shared MFs (which together form $n_{2}$ ) with one of the end MFs making up $n_{1}$ or $n_{3}$. In contrast, the other braiding operations, which involve exchange of MFs within each qubit, merely result in single-qubit rotations.

Thus, a non-trivial (entangling) two-qubit gate is e.g., obtained by the combined operations $M_{1} M_{3} P_{F}^{1} P_{F}^{2} B_{2} P_{E}^{1} P_{E}^{2} I_{1} I_{2} I_{3}$ (initialize Majorana number states, empty dots, braid MFs 2 and 3, fill dots, measure $n_{1}$ and $n_{3}$ ). By combinations of braiding operations we can construct e.g., a CNOT gate $\frac{40}{}$ acting on the topological two-qubit system. Applying the CNOT gate to the topological part of the state $|j\rangle$ in Eq. (15) before filling the dots gives

$$
\begin{aligned}
P_{F}^{1} P_{F}^{2} \mathrm{CNOT}|j\rangle & =-P_{F}^{1} P_{F}^{2}|00\rangle_{D}|\overline{1} \overline{1}\rangle_{Q} \\
& =-\frac{1}{2}\left(|\uparrow \uparrow\rangle_{D}|\overline{0} \overline{1}\rangle_{Q}-|\downarrow \downarrow\rangle_{D}|\overline{1} \overline{0}\rangle_{Q}\right. \\
& \left.+i|\downarrow \uparrow\rangle_{D}|\overline{1} \overline{1}\rangle_{Q}-i|\uparrow \downarrow\rangle_{D}|\overline{0} \overline{0}\rangle_{Q}\right) .(17
\end{aligned}
$$

After measuring $n_{1}$ and $n_{3}$ we are of course still left with four possible outcomes; if $n_{1}=n_{3}=0$ is measured a CNOT gate has been performed on the spin qubits, while other measured $n_{1}$ and $n_{3}$ correspond to other gates. However, as was briefly mentioned above, these other gates are related to the $|\overline{0} \overline{0}\rangle_{Q}$-gate by single spin-qubit rotations. It holds in general that to retrieve the result of the $|\overline{0} \overline{0}\rangle_{Q}$-gate (up to a phase factor), one should perform a $\pi$-rotation around the $y$-axis of the spin on dot 1 if $n_{1}=1$ and on dot 2 if $n_{3}=1$. Thus, in combination with the parity measurements, (possibly) followed by single-qubit rotations, our proposed two-qubit spin gates are fully deterministic. The result in Eq. (17) may seem trivial, but starting from the general two-qubit state $|i\rangle$ rather than $\left|i^{\prime}\right\rangle$, one sees that this procedure indeed produces a CNOT gate, up to single-qubit rotations.

\section{PHYSICAL ASSUMPTIONS AND SOURCES OF ERRORS}

Finally, we discuss the assumptions made when writing down the model in Eq. (1), as well as the effect on the results when the most crucial assumptions are not perfectly fulfilled.

First, we have included only a single level on each dot and in addition let $U_{i} \rightarrow \infty$. The likely most experimentally attractive option is to construct the quantum dots from the same 1D wires as the TS, but not covered by a superconductor and separated from the TS for example by electric gates. The dots would then be defined in e.g., InAs or InSb nanowires (good candidates for 1D TS). Such dots have been found to exhibit level spacings and Coulomb charging energies of several meV, as discussed e.g., in Refs. 41, 42. Since this is much larger than both the expected TS gap $(\lesssim 1 \mathrm{meV})$ and operating temperature $(\lesssim 100 \mathrm{mK})$, these assumptions are rather unproblematic.

Second, the dots are assumed to be spin degenerate. This could be achieved if the Zeeman term needed to induce topological superconductivity is provided by proximity with a magnetic insulator, see e.g., Refs. 17, 18, rather than induced by an external magnetic field. Alternatively, a magnetic field could be tuned to recover spin degeneracy, but between spin-up and spin-down states in different dot orbitals. It has also been suggested 43 that strong interactions may lower the magnetic field needed to produce Majorana fermions, or even cause them to appear at zero field due to spontaneous breaking of timereversal symmetry. Finally, the $g$-factor on the dot can be much smaller than that in the TS (even if they are made from the same material since strong confinement often renormalizes the $g$-factor), making it possible to reach the topological regime while introducing only a small Zeeman splitting on the dot. In fact, a small Zeeman splitting, $\delta B \ll \lambda$, is acceptable. A gate sweep which is adiabatic with respect to $\lambda$, but non-adiabatic with respect to $\delta B$, then still results in the same filling and emptying operators, Eqs. (40) and (5), see Ref. 28.

Finally, we have assumed perfect anti-parallel spin polarizations of the two MFs which couple to a given dot. Under idealized conditions, for some realizations of MFs, the spin polarization direction is controlled by the directionality of the border between topological and nontopological regions and would therefore naturally be opposite for opposite edges as sketched in Fig. 1, see e.g., Refs. 17, 20, 44, 45. Under non-ideal conditions, the polarization direction will depend on the details of the edge, as discussed in some detail e.g., in Refs. 46, 47. Tuning the MF spin polarization can e.g., be achieved through control of the chemical potential close to the edge, which must likely be experimentally controllable to reach the 
topological regime anyway. If the dot level is tuned close to resonance, a deviation from anti-parallel polarizations lead to a finite supercurrent through the system, which can therefore be used as an indicator when fine tuning to the anti-parallel configuration.

If perfect anti-parallel spin polarizations cannot be achieved, all is not lost. The supercurrent and the associated splitting of the TS ground state degeneracy can be eliminated by control of the relative phase difference between the TS on the two sides of the $\operatorname{dot}^{\underline{34}}$. Filling and emptying dot $i$ is then described by the operators [cf. Eqs. (4) and (50)]

$$
\begin{aligned}
\tilde{P}_{F}^{i} & =\frac{1}{\sqrt{2}}\left[\gamma_{2 i}\left(d_{i \uparrow}^{\dagger} \cos \frac{\theta}{4}+d_{i \downarrow}^{\dagger} \sin \frac{\theta}{4}\right)\right. \\
& \left.+\quad \gamma_{2 i+1}\left(d_{i \downarrow}^{\dagger} \cos \frac{\theta}{4}+d_{i \uparrow}^{\dagger} \sin \frac{\theta}{4}\right)\right], \\
\tilde{P}_{E}^{i} & =N\left[\gamma_{2 i}\left(d_{i \uparrow} \cos \frac{\theta}{4}+d_{i \downarrow} \sin \frac{\theta}{4}\right)\right. \\
& \left.+\quad \gamma_{2 i+1}\left(d_{i \downarrow} \cos \frac{\theta}{4}+d_{i \uparrow} \sin \frac{\theta}{4}\right)\right],
\end{aligned}
$$

where $\theta$ is the angular deviation from perfect anti-parallel spin polarizations. Acting with $\tilde{P}_{F}^{i}$ or $\tilde{P}_{E}^{i}$ still accomplishes a quantum information transfer between topological qubits and spin qubits, but introduces an error $\delta=\sin \frac{\theta}{4}$ (compare with the ideal cases described by Eqs. (5) and (9) in Ref. 28). The two-qubit gates discussed above require only two empty and two fill operations. At least for a proof of principle experiment, a rather sizable spin mis-alignment is therefore acceptable.

\section{CONCLUSIONS}

The setup in Fig. 1 1 could easily be extended to include an arbitrary number of quantum dots coupled to edge
Majorana bound states. In such a geometry, the combined results described here and in Ref. 28 allow transferring spin qubits between arbitrary dots, initializing any given pair of spin qubits in a maximally entangled Bell state, and performing non-trivial two-qubit gates on arbitrary spin qubits. Furthermore, the interface with the topological qubits provides a quantum memory with potentially very long coherence times. Together with the fast manipulation of single spin qubits or spatially close qubit pairs, known from conventional spin qubit systems, this provides an interesting platform for quantum computation with spins.

In addition to the possibility of acting on spatially separated spin qubits, our proposed topologically-assisted two-qubit gate has the advantage of avoiding any continuous errors. The braiding-, transfer-, and measurement operations can be done to yield exactly the desired operation, unlike a controlled exchange interaction which requires very accurate timing and unavoidably introduces small errors. While the braiding operations are being performed, both dots are empty and all information is stored in the topological system and therefore benefits from the associated (hopefully) long coherence times. Only the transfer operations are unprotected, but, assuming that the braiding operations can be carried out without decoherence, we have reduced the problem of performing a two-spin-qubit gate to that of gatecontrolled transfer of two individual charges.

Note that the problem of generalizing such an overlapping qubit system to more than two qubits 39,40 is not an issue in our case, since at any given time, at the most two spin qubits have to be stored in the topological system, and any other spin-qubits (on other dots) are of course unaffected by the braiding and transfer operations done to these qubits.
1 D. Loss and D. P. Di Vincenzo, Phys. Rev. A 57, 120 (1998)

${ }^{2}$ F. H. L. Koppens, J. A. Folk, J. M. Elzerman, R. Hanson, L. H. W. van Beveren, I. T. Vink, H. P. Tranitz, W. Wegscheider, L. P. Kouwenhoven, and L. M. K. Vandersypen, Science 309, 1346 (2005)

3 J. R. Petta, A. C. Johnson, J. M. Taylor, E. A. Laird, A. Yacoby, M. D. Lukin, C. M. Marcus, M. P. Hanson, and A. C. Gossard, Science 309, 2180 (2005)

${ }^{4}$ F. H. L. Koppens, C. Buizert, K. J. Tielrooij, I. T. Vink, K. C. Nowack, T. Meunier, L. P. Kouwenhoven, and L. M. K. Vandersypen, Nature 442, 766 (2006)

${ }^{5}$ K. C. Nowack, F. H. L. Koppens, Y. V. Nazarov, and L. M. K. Vandersypen, Science 318, 430 (2007)

6 D. P. DiVincenzo, D. Bacon, J. Kempe, G. Burkard, and K. B. Whaley, Nature 408, 339 (2000)

7 J. M. Elzerman, R. Hanson, L. H. W. van Beveren, B. Witkamp, and L. M. K. V. L. P. K. and, Nature 430, 431 (2004)
${ }^{8}$ K. M. Svore, B. M. Terhal, and D. P. DiVincenzo, Phys. Rev. A 72, 022317 (2005)

9 J. I. Cirac and P. Zoller, Phys. Rev. Lett. 74, 4091 (1995)

10 M. A. Sillanpää, J. I. Park, and R. W. Simmonds, Nature 449, 438 (2007)

11 J. Majer, J. M. Chow, J. M. Gambetta, J. Koch, B. R. J. J. A. Schreier, L. Frunzio, D. I. Schuster, A. A. Houck, A. Wallraff, A. Blais, M. H. Devoret, S. M. Girvin, and R. J. Schoelkopf, Nature 449, 443 (2007)

12 A. Imamoglu, D. D. Awschalom, G. Burkard, D. P. DiVincenzo, D. Loss, M. Sherwin, and A. Small, Phys. Rev. Lett. 83, 4204 (1999)

13 L. Childress, A. S. Sørensen, and M. D. Lukin, Phys. Rev. A 69, 042302 (2004)

14 P.-Q. Jin, M. Marthaler, A. Shnirman, and G. Schön, Phys. Rev. Lett. 108, 190506 (2012)

15 M. Friesen, A. Biswas, X. Hu, and D. Lidar, Phys. Rev. Lett. 98, 230503 (2007) 
16 S. Das Sarma, C. Nayak, and S. Tewari, Phys. Rev. B 73, 220502(R) (2006)

17 L. Fu and C. L. Kane, Phys. Rev. Lett. 100, 096407 (2008)

18 J. D. Sau, R. M. Lutchyn, S. Tewari, and S. Das Sarma, Phys. Rev. Lett. 104, 040502 (2010)

19 J. Alicea, Phys. Rev. B 81, 125318 (2010)

20 Y. Oreg, G. Refael, and F. von Oppen, Phys. Rev. Lett. 105, $177002(2010)$

21 R. M. Lutchyn, J. D. Sau, and S. Das Sarma, Phys. Rev. Lett. 105, 077001 (2010)

${ }^{22}$ F. Wilczek, Nature Physics 5, 614 (2009)

${ }^{23}$ G. Moore and N. Read, Nucl. Phys. B 360, 362 (1991)

24 A. Y. Kitaev, Ann. Phys. 303, 2 (2003)

25 C. Nayak, S. H. Simon, A. Stern, M. Freedman, and S. Das Sarma, Rev. Mod. Phys. 80, 1083 (2008)

26 A. Stern, Nature 464, 187 (2010)

27 D. A. Ivanov, Phys. Rev. Lett. 86, 268 (2001)

28 M. Leijnse and K. Flensberg, Phys. Rev. Lett. 107, 210502 (2011)

29 F. Hassler, A. R. Akhmerov, C.-Y. Hou, and C. W. J. Beenakker, New Journal of Physics 12, 125002 (2010)

30 J. D. Sau, S. Tewari, and S. Das Sarma, Phys. Rev. A 82, $052322(2010)$

31 L. Jiang, C. L. Kane, and J. Preskill, Phys. Rev. Lett. 106, 130504 (2011)

32 P. Bonderson and R. M. Lutchyn, Phys. Rev. Lett. 106, 130505 (2011)

33 M. Leijnse and K. Flensberg, Phys. Rev. B 84, 140501(R)
(2011)

34 K. Flensberg, Phys. Rev. Lett. 106, 090503 (2011)

35 J. Alicea, Y. Oreg, G. Refael, F. von Oppen, and M. P. A. Fisher, Nature Physics 7, 412 (2011)

${ }^{36}$ B. van Heck, A. Akhmerov, F. Hassler, M. Burrello, and C. W. J. Beenakker, New Journal of Physics 14, 035019 (2012)

37 B. I. Halperin, Y. Oreg, A. Stern, G. Refael, J. Alicea, and F. von Oppen, Phys. Rev. B 85, 144501 (2012)

38 W. Bishara, P. Bonderson, C. Nayak, K. Shtengel, and J. K. Slingerland, Phys. Rev. B 80, 155303 (2009)

39 S. Bravyi, Phys. Rev. A 73, 042313 (2006)

40 L. S. Georgiev, Phys. Rev. B 74, 235112 (2006)

41 C. Fasth, A. Fuhrer, L. Samuelson, V. N. Golovach, and D. Loss, Phys. Rev. Lett. 98, 266801 (2007)

${ }^{42}$ H. A. Nilsson, P. Caroff, C. Thelander, M. Larsson, J. B. Wagner, L.-E. Wernersson, L. Samuelson, and H. Q. Xu, Nano Lett. 9, 3151 (2009)

43 E. M. Stoudenmire, J. Alicea, O. A. Starykh, and M. P. A. Fisher, Phys. Rev. B 84, 014503 (2011)

44 L. Fu and C. L. Kane, Phys. Rev. B 79, 161408(R) (2009)

${ }^{45}$ V. Shivamoggi, G. Refael, and J. E. Moore, Phys. Rev. B 82, 041405(R) (2010)

46 M. Kjaergaard, K. Wölms, and K. Flensberg, Phys. Rev. B 85, 020503(R) (2012)

47 D. Sticlet, C. Bena, and P. Simon, Phys. Rev. Lett. 108, $096802(2012)$ 